\title{
Correlation Function and Simplified TBA Equations for XXZ Chain*
}

\author{
Minoru TAKAHASHI
}

Fachbereich C Physik, Bergische Universität Wuppertal, 42097 Wuppertal, Germany

E-mail:mtaka@issp.u-tokyo.ac.jp

Received September 27, 2010, in final form December 27, 2010; Published online January 08, 2011 doi:10.3842/SIGMA.2011.004

\begin{abstract}
The calculation of the correlation functions of Bethe ansatz solvable models is very difficult problem. Among these solvable models spin 1/2 XXX chain has been investigated for a long time. Even for this model only the nearest neighbor and the second neighbor correlations were known. In 1990's multiple integral formula for the general correlations is derived. But the integration of this formula is also very difficult problem. Recently these integrals are decomposed to products of one dimensional integrals and at zero temperature, zero magnetic field and isotropic case, correlation functions are expressed by $\log 2$ and Riemann's zeta functions with odd integer argument $\zeta(3), \zeta(5), \zeta(7), \ldots$ We can calculate density sub-matrix of successive seven sites. Entanglement entropy of seven sites is calculated. These methods can be extended to XXZ chain up to $n=4$. Correlation functions are expressed by the generalized zeta functions. Several years ago I derived new thermodynamic Bethe ansatz equation for XXZ chain. This is quite different with Yang-Yang type TBA equations and contains only one unknown function. This equation is very useful to get the high temperature expansion. In this paper we get the analytic solution of this equation at $\Delta=0$.
\end{abstract}

Key words: thermodynamic Bethe ansatz equation; correlation function

2010 Mathematics Subject Classification: 16T25; 17B37; 82B23

\section{Introduction}

We consider the spin $1 / 2 \mathrm{XXZ}$ chain

$$
\mathcal{H}=-J \sum_{l=1}^{N} S_{l}^{x} S_{l+1}^{x}+S_{l}^{y} S_{l+1}^{y}+\Delta\left(S_{l}^{z} S_{l+1}^{z}-\frac{1}{4}\right)-2 h \sum_{l=1}^{N} S_{l}^{z} .
$$

Among the solvable models this model has been investigated for a long time.

It was believed that the exact calculation of correlation function is impossible except the nearest neighbor correlation function. I derived the second neighbor correlation function for $J<0, T=0, h=0, \Delta=1$ using the Lieb-Wu solution of one-dimensional Hubbard model [1]. Details are given in Appendix A. In 1990's multiple integral formula were proposed for $h=0$, $T=0$ and recently multiple integral formula was extended to $h \neq 0, T \neq 0$. But the factorization of these multiple integrals to the integrals of lower dimension still remains a difficult problem. I explain the present situation of factorization in Section 2.

Since Yang and Yang proposed the thermodynamic Bethe ansatz (TBA) equation for one dimensional bosons [2], the calculation of free energy becomes important for other solvable models. Yang-Yang type integral equations for (1) were proposed in early 1970's [3, 4, 5]. In this theory infinite number of unknown functions appeared for $|\Delta| \geq 1$ and finite number of

\footnotetext{
* This paper is a contribution to the Proceedings of the International Workshop "Recent Advances in Quantum Integrable Systems". The full collection is available at http://www.emis.de/journals/SIGMA/RAQIS2010.html
} 
unknowns appear for $\Delta=\cos \pi \nu$ with $\nu=$ rational number. The equations change by the value of $\Delta$ and not so convenient for numerical calculations. But some important physical properties at low temperature were investigated using these equations.

Around 1990 the quantum transfer matrix method was applied to this model and numerical results coincide with those of Yang-Yang type equations $[6,7,8]$. In 2001 I proposed a new TBA equation which contains only one unknown function for (1) [9, 10]. This equation is very convenient to do the high-temperature expansions and we get one hundred-th order of high temperature expansion [11]. The traditional cluster expansion method gives up to 22'nd. This method was also applied to the Perk-Schultz model [12].

Unfortunately this equation does not numerically converge at low temperature like $T /|J|<$ 0.07 because the integrand strongly oscillates. Some other numerical method is necessary. I showed that this equation gives the known exact results in Ising limit $(\Delta \rightarrow \infty$ and $J \Delta=$ finite) [9]. In Section 3 I give the analytic solution of this equation for XY case $(\Delta=0)$.

\section{Correlation functions of XXZ chain}

\subsection{Known exact results for $J<0, T=0, h=0, \Delta=1$}

1. Nearest-neighbor correlator

$$
\left\langle S_{j}^{z} S_{j+1}^{z}\right\rangle=\frac{1}{12}-\frac{1}{3} \ln 2=-0.1477157268 \ldots
$$

from the ground state energy per site by Hulthén [13] (1938).

2. Next nearest-neighbor correlator for XXX

$$
\left\langle S_{j}^{z} S_{j+2}^{z}\right\rangle=\frac{1}{12}-\frac{4}{3} \ln 2+\frac{3}{4} \zeta(3)=0.06067976995 \ldots,
$$

from the ground state energy of the half-filled Hubbard model by Takahashi [1] (1977).

3. The twisted four-body correlation function

$$
\left\langle\left(\boldsymbol{S}_{j} \times \boldsymbol{S}_{j+1}\right) \cdot\left(\boldsymbol{S}_{j+2} \times \boldsymbol{S}_{j+3}\right)\right\rangle=\frac{1}{2} \ln 2-\frac{3}{8} \zeta(3)=-0.104197748
$$

from the third derivative of transfer matrix by Muramoto and Takahashi [14] (1999).

\subsection{Multiple-integral representations for density matrix}

For more general XXZ model with an anisotropy parameter $\Delta$

1. $\Delta>1, T=0, h=0$ : Vertex operator approach $U_{q}(\hat{s l}(2))$ Jimbo, Miki, Miwa, Nakayashiki $[15,16](1992)$.

2. $-1<\Delta<1, T=0, h=0$ : qKZ equation Jimbo and Miwa [17] (1996).

3. Rederivation by the quantum inverse scattering method. Generalization to the XXZ model with a magnetic field, $T=0$ Kitanine, Maillet, Terras [18] (1998).

4. Integral formula for finite temperature and finite magnetic field Göhmann, Klümper, Seel [19] (2004).

Correlation function for successive elementary block

$$
\rho_{n}\left(\left\{\epsilon_{j}, \epsilon_{j}^{\prime}\right\}\right)=\frac{\left\langle\psi\left|\prod_{j=1}^{n} E_{j}^{\epsilon_{j}^{\prime}, \epsilon_{j}}\right| \psi\right\rangle}{\langle\psi \mid \psi\rangle},
$$




$$
E_{j}^{+,+}=\left(\begin{array}{ll}
1 & 0 \\
0 & 0
\end{array}\right)_{[j]}, \quad E_{j}^{+,-}=\left(\begin{array}{ll}
0 & 1 \\
0 & 0
\end{array}\right)_{[j]}, \quad E_{j}^{-,+}=\left(\begin{array}{cc}
0 & 0 \\
1 & 0
\end{array}\right)_{[j]}, \quad E_{j}^{-,-}=\left(\begin{array}{cc}
0 & 0 \\
0 & 1
\end{array}\right)_{[j]},
$$

is represented by $n$-fold integral. For example the emptiness formation probability (EFP) for $\mathrm{XXX}$ chain at $T=h=0$ is

$$
\begin{aligned}
P(n) & \equiv\left\langle\left(S_{1}^{z}+\frac{1}{2}\right)\left(S_{2}^{z}+\frac{1}{2}\right) \cdots\left(S_{n}^{z}+\frac{1}{2}\right)\right\rangle=\rho_{n++\cdots+}^{++\cdots+} \\
& =(-\pi)^{\frac{n(n-1)}{2}} 2^{-n} \int_{-\infty}^{\infty} \mathrm{d}^{n} \lambda \prod_{a>b}^{n} \frac{\sinh \pi\left(\lambda_{a}-\lambda_{b}\right)}{\lambda_{a}-\lambda_{b}-\mathrm{i}} \prod_{j=1}^{n} \frac{\left(\lambda_{j}-\frac{\mathrm{i}}{2}\right)^{j-1}\left(\lambda_{j}+\frac{\mathrm{i}}{2}\right)^{n-j}}{\cosh ^{n} \pi \lambda_{j}} .
\end{aligned}
$$

Other arbitrary correlation functions over successive $n$-sites have similar $n$-fold integral representation.

\subsection{Boos-Korepin method to evaluate integrals}

Here I introduce the details of direct factorization of multiple integrals by Boos and Korepin $[20,21]$.

1. Transform the integrand to a certain canonical form without changing the integral value.

2. Perform the integration using the residue theorem.

Example:

$$
\begin{aligned}
& P(3) \equiv \prod_{j=1}^{3} \int_{-\infty-\frac{i}{2}}^{\infty-\frac{i}{2}} \frac{\mathrm{d} \lambda_{j}}{2 \pi i} U_{3}\left(\lambda_{1}, \lambda_{2}, \lambda_{3}\right) T_{3}\left(\lambda_{1}, \lambda_{2}, \lambda_{3}\right), \\
& U_{3}\left(\lambda_{1}, \lambda_{2}, \lambda_{3}\right) \equiv \pi^{6} \frac{\prod_{1 \leq k<j \leq 3} \sinh \pi\left(\lambda_{j}-\lambda_{k}\right)}{\prod_{j=1}^{3} \sinh ^{3} \pi \lambda_{j}}, \\
& T_{3}\left(\lambda_{1}, \lambda_{2}, \lambda_{3}\right) \equiv \frac{\left(\lambda_{1}+i\right)^{2} \lambda_{2}\left(\lambda_{2}+i\right) \lambda_{3}^{2}}{\left(\lambda_{2}-\lambda_{1}-i\right)\left(\lambda_{3}-\lambda_{1}-i\right)\left(\lambda_{3}-\lambda_{2}-i\right)} .
\end{aligned}
$$

Details of transformation are given in Appendix B.

In canonical form denominator is

$$
\prod_{k=1}^{l}\left(\lambda_{2 k-1}-\lambda_{2 k}\right), \quad l \leq[n / 2]
$$

The $n$-dimensional integral is decomposed to one and two dimensional integrals. Transform $T_{3}\left(\lambda_{1}, \lambda_{2}, \lambda_{3}\right)$ into a canonical form $T_{3}^{\mathrm{c}}\left(\lambda_{1}, \lambda_{2}, \lambda_{3}\right)$

$$
\begin{aligned}
& T_{3}\left(\lambda_{1}, \lambda_{2}, \lambda_{3}\right) \sim T_{3}^{\mathrm{c}}\left(\lambda_{1}, \lambda_{2}, \lambda_{3}\right)=P_{0}^{(3)}+\frac{P_{1}^{(3)}}{\lambda_{2}-\lambda_{1}}, \\
& P_{0}^{(3)}=-2 \lambda_{2} \lambda_{3}^{2}, \quad P_{1}^{(3)}=\frac{1}{3}-i \lambda_{1}-i \lambda_{3}-2 \lambda_{1} \lambda_{3} .
\end{aligned}
$$

Perform the integration

$$
J_{0}^{(3)}=\prod_{j=1}^{3} \int_{-\infty-\frac{i}{2}}^{\infty-\frac{i}{2}} \frac{\mathrm{d} \lambda_{j}}{2 \pi i} U_{3}\left(\lambda_{1}, \lambda_{2}, \lambda_{3}\right) P_{0}^{(3)}=\frac{1}{4}
$$




$$
\begin{aligned}
& J_{1}^{(3)}=\prod_{j=1}^{3} \int_{-\infty-\frac{i}{2}}^{\infty-\frac{i}{2}} \frac{\mathrm{d} \lambda_{j}}{2 \pi i} U_{3}\left(\lambda_{1}, \lambda_{2}, \lambda_{3}\right) \frac{P_{1}^{(3)}}{\lambda_{2}-\lambda_{1}}=-\ln 2+\frac{3}{8} \zeta(3), \\
& P(3)=J_{0}^{(3)}+J_{1}^{(3)}=\frac{1}{4}-\ln 2+\frac{3}{8} \zeta(3) .
\end{aligned}
$$

Thus second neighbor correlator was rederived from the integral formula.

For $P(4)=\rho_{4++++}^{++++}$the integrand is

$$
\frac{\left(\lambda_{1}+i\right)^{3}\left(\lambda_{2}+i\right)^{2} \lambda_{2}\left(\lambda_{3}+i\right) \lambda_{3}^{2} \lambda_{4}^{3}}{\left(\lambda_{43}-i\right)\left(\lambda_{42}-i\right)\left(\lambda_{41}-i\right)\left(\lambda_{32}-i\right)\left(\lambda_{31}-i\right)\left(\lambda_{21}-i\right)} .
$$

Here we put $\lambda_{a b} \equiv \lambda_{a}-\lambda_{b}$. Canonical form is $P_{0}^{(4)}+P_{1}^{(4)} / \lambda_{21}+P_{2}^{(4)} /\left(\lambda_{21} \lambda_{43}\right)$,

$$
\begin{aligned}
P_{0}^{(4)}= & -\frac{34}{5} \lambda_{2} \lambda_{3}^{2} \lambda_{4}^{3}, \\
P_{1}^{(4)}= & \lambda_{1}^{2}\left(30 \lambda_{3}^{2} \lambda_{4}^{3}+30 i \lambda_{3} \lambda_{4}^{3}-16 \lambda_{4}^{3}+18 \lambda_{3} \lambda_{4}^{2}+8 \lambda_{4}\right) \\
& +\lambda_{1}\left(30 i \lambda_{3}^{2} \lambda_{4}^{3}+30 \lambda_{3} \lambda_{4}^{3}-16 i \lambda_{4}^{3}+18 i \lambda_{3} \lambda_{4}^{2}-4 \lambda_{4}^{2}+4 i \lambda_{4}\right) \\
& -20 \lambda_{3}^{2} \lambda_{4}^{3}-20 i \lambda_{3} \lambda_{4}^{3}+\frac{54}{5} \lambda_{4}^{3}-\frac{42}{5} \lambda_{3} \lambda_{4}^{2}-\frac{43}{10} i \lambda_{4}, \\
P_{2}^{(4)}= & 2 \lambda_{1}^{2} \lambda_{3}^{2}+4 i \lambda_{1} \lambda_{3}^{2}-\frac{3}{2} \lambda_{3}^{2}-\frac{3}{2} \lambda_{1} \lambda_{3}-i \lambda_{3}+\frac{1}{5}, \\
P(4)= & \frac{1}{5}-2 \ln 2+\frac{173}{60} \zeta(3)-\frac{11}{6} \ln 2 \cdot \zeta(3)-\frac{51}{80} \zeta^{2}(3)-\frac{55}{24} \zeta(5)+\frac{85}{24} \ln 2 \cdot \zeta(5) .
\end{aligned}
$$

In 2003, we calculated $\rho_{+-+-}^{+-+-}$by Boos-Korepin method and obtained all the correlation functions on 4 lattice sites [22]. Especially, the third-neighbor correlator is

$$
\begin{aligned}
\left\langle S_{j}^{z} S_{j+3}^{z}\right\rangle & =\frac{1}{12}-3 \ln 2+\frac{37}{6} \zeta(3)-\frac{14}{3} \ln 2 \cdot \zeta(3)-\frac{3}{2} \zeta(3)^{2}-\frac{125}{24} \zeta(5)+\frac{25}{3} \ln 2 \cdot \zeta(5) \\
& =-0.05024862725 \ldots
\end{aligned}
$$

The other correlation functions for $n=4$ are

$$
\begin{aligned}
\left\langle S_{j}^{x} S_{j+1}^{x} S_{j+2}^{z} S_{j+3}^{z}\right\rangle= & \frac{1}{240}+\frac{1}{12} \ln 2-\frac{91}{240} \zeta(3)+\frac{1}{6} \ln 2 \cdot \zeta(3) \\
& +\frac{3}{80} \zeta(3)^{2}+\frac{35}{96} \zeta(5)-\frac{5}{24} \ln 2 \cdot \zeta(5), \\
\left\langle S_{j}^{x} S_{j+1}^{z} S_{j+2}^{x} S_{j+3}^{z}\right\rangle= & \frac{1}{240}-\frac{1}{6} \ln 2+\frac{77}{120} \zeta(3)-\frac{5}{12} \ln 2 \cdot \zeta(3) \\
& -\frac{3}{20} \zeta(3)^{2}-\frac{65}{96} \zeta(5)+\frac{5}{6} \ln 2 \cdot \zeta(5), \\
\left\langle S_{j}^{x} S_{j+1}^{z} S_{j+2}^{z} S_{j+3}^{x}\right\rangle= & \frac{1}{240}-\frac{1}{4} \ln 2+\frac{169}{240} \zeta(3)-\frac{5}{12} \ln 2 \cdot \zeta(3) \\
& -\frac{3}{20} \zeta(3)^{2}-\frac{65}{96} \zeta(5)+\frac{5}{6} \ln 2 \cdot \zeta(5), \\
\left\langle S_{j}^{z} S_{j+1}^{z} S_{j+2}^{z} S_{j+3}^{z}\right\rangle= & \left\langle S_{j}^{x} S_{j+1}^{x} S_{j+2}^{z} S_{j+3}^{z}\right\rangle+\left\langle S_{j}^{x} S_{j+1}^{z} S_{j+2}^{x} S_{j+3}^{z}\right\rangle+\left\langle S_{j}^{x} S_{j+1}^{z} S_{j+2}^{z} S_{j+3}^{x}\right\rangle .
\end{aligned}
$$

From these results we can reproduce the twisted correlation function in (4).

In a similar way, $P(5)$ was calculated after very tedious calculations [23]

$$
\begin{aligned}
P(5)= & \frac{1}{6}-\frac{10}{3} \ln 2+\frac{281}{24} \zeta(3)-\frac{45}{2} \ln 2 \cdot \zeta(3)-\frac{489}{16} \zeta(3)^{2}-\frac{6775}{192} \zeta(5)+\frac{1225}{6} \ln 2 \cdot \zeta(5) \\
& -\frac{425}{64} \zeta(3) \cdot \zeta(5)-\frac{12125}{256} \zeta(5)^{2}+\frac{6223}{256} \zeta(7)-\frac{11515}{64} \ln 2 \cdot \zeta(7)+\frac{42777}{512} \zeta(3) \cdot \zeta(7) .
\end{aligned}
$$

But the direct integral of other correlations for five sites is almost impossible. 


\subsection{Algebraic approach and $\mathrm{qKZ}$ relation}

Next problem is to calculate $\left\langle S_{j}^{z} S_{j+4}^{z}\right\rangle$ for XXX model. In principle, it's possible to calculate other five-dimensional integrals by use of Boos-Korepin method. It, however, will take tremendous amount of time.

We propose a different method ("algebraic approach") and obtain analytical form of $\left\langle S_{j}^{z} S_{j+4}^{z}\right\rangle$. This is a generalization of the method by Boos, Korepin, Smirnov (2003) for $P(6)$ [24]. We consider the density matrix $\rho$ of successive $n$ sites of inhomogeneous six vertex model with different spectral parameter $z_{j}$ for $j$-th site

$$
\begin{aligned}
& \lim _{z_{i} \rightarrow 0} \rho_{n, \epsilon_{1}, \ldots, \epsilon_{n}}^{\epsilon_{1}^{\prime}, \ldots, \epsilon_{n}^{\prime}}\left(z_{1}, z_{2}, \ldots, z_{n}\right)=\left\langle E_{\epsilon_{1}}^{\epsilon_{1}^{\prime}} \cdots E_{\epsilon_{n}}^{\epsilon_{n}^{\prime}}\right\rangle, \\
& \left(E_{\epsilon}^{\epsilon^{\prime}}\right)_{s, s^{\prime}}=\delta_{\epsilon, s} \delta_{\epsilon^{\prime}, s^{\prime}}, \quad \epsilon, \epsilon^{\prime}= \pm 1 .
\end{aligned}
$$

For $n=1$, and 2 we have

$$
\begin{aligned}
& \rho_{1,+}^{+}\left(z_{1}\right)=\rho_{1,-}^{-}\left(z_{1}\right)=\frac{1}{2}, \quad \rho_{1,+}^{-}\left(z_{1}\right)=\rho_{1,-}^{+}\left(z_{1}\right)=0, \\
& \rho_{2,++}^{++}\left(z_{1}, z_{2}\right)=\frac{1}{4}+\frac{1}{6} \omega\left(z_{1}-z_{2}\right), \\
& \rho_{2,+-}^{+-}\left(z_{1}, z_{2}\right)=-\frac{1}{6} \omega\left(z_{1}-z_{2}\right), \quad \rho_{2,+-}^{-+}\left(z_{1}, z_{2}\right)=\frac{1}{3} \omega\left(z_{1}-z_{2}\right),
\end{aligned}
$$

with

$$
\begin{aligned}
& \omega(x) \equiv \frac{1}{2}+2 \sum_{k=1}^{\infty}(-1)^{k} k \frac{1-x^{2}}{k^{2}-x^{2}}=\frac{1}{2}-2\left(1-x^{2}\right) \sum_{k=0}^{\infty} x^{2 k} \zeta_{a}(2 k+1), \\
& \zeta_{a}(x) \equiv \sum_{n=1}^{\infty}(-1)^{n-1} n^{-x}=\left(1-2^{1-x}\right) \zeta(x), \quad \zeta_{a}(1)=\ln 2, \\
& \omega(x+1)=-\frac{x(x+2)}{x^{2}-1} \omega(x)-\frac{3}{2} \frac{1}{1-x^{2}}, \quad \omega(-x)=\omega(x), \quad \omega( \pm i \infty)=0 .
\end{aligned}
$$

The general element of density matrix must satisfy the following algebraic relations.

- Translational invariance

$$
\rho_{n, \epsilon_{1}, \ldots, \epsilon_{n}}^{\epsilon_{1}^{\prime}, \ldots, \epsilon_{n}^{\prime}}\left(z_{1}+x, \ldots, z_{n}+x\right)=\rho_{n, \epsilon_{1}, \ldots, \epsilon_{n}}^{\epsilon_{1}^{\prime}, \ldots, \epsilon_{n}^{\prime}}\left(z_{1}, \ldots, z_{n}\right) .
$$

- Transposition, negating and reverse-order relations

$$
\begin{aligned}
\rho_{n, \epsilon_{1}, \ldots, \epsilon_{n}}^{\epsilon_{1}^{\prime}, \ldots, \epsilon_{n}^{\prime}}\left(z_{1}, \ldots, z_{n}\right) & =\rho_{n, \epsilon_{1}^{\prime}, \ldots, \epsilon_{n}^{\prime}}^{\epsilon_{1}, \ldots, \epsilon_{n}}\left(-z_{1}, \ldots,-z_{n}\right) \\
& =\rho_{n,-\epsilon_{1}, \ldots,-\epsilon_{n}}^{-\epsilon_{1}^{\prime}, \ldots,-\epsilon_{n}^{\prime}}\left(z_{1}, \ldots, z_{n}\right)=\rho_{n, \epsilon_{n}, \ldots, \epsilon_{1}}^{\epsilon_{n}^{\prime}, \ldots, \epsilon_{1}^{\prime}}\left(-z_{n}, \ldots,-z_{1}\right) .
\end{aligned}
$$

- Intertwining relation

$$
\begin{aligned}
& R_{\tilde{\epsilon}_{j}^{\prime} \epsilon_{j+1}^{\prime}}^{\epsilon_{j}^{\prime} \epsilon_{j+1}^{\prime}}\left(z_{j}-z_{j+1}\right) \rho_{\ldots . \ldots \epsilon_{j+1}, \epsilon_{j} \ldots}^{\ldots \tilde{\epsilon}_{j+1}^{\prime}, \tilde{\epsilon}_{j}^{\prime} \ldots}\left(\ldots z_{j+1}, z_{j} \ldots\right) \\
& \quad=\rho_{\ldots \epsilon_{j}, \epsilon_{j}, \epsilon_{j+1}^{\prime} \ldots}^{\prime}\left(\ldots z_{j}, z_{j+1} \ldots\right) R_{\epsilon_{j} \epsilon_{j+1}}^{\tilde{\epsilon}_{j} \tilde{\epsilon}_{j+1}}\left(z_{j}-z_{j+1}\right), \\
& R_{++}^{++}(z)=R_{--}^{--}(z)=1, \quad R_{+-}^{+-}(z)=R_{-+}^{-+}(z)=\frac{z}{z+1}, \\
& R_{-+}^{+-}(z)=R_{+-}^{-+}(z)=\frac{1}{z+1} .
\end{aligned}
$$


- Reduction relation

$$
\rho_{n,+, \epsilon_{2}, \ldots, \epsilon_{n}}^{+, \epsilon_{2}^{\prime}, \ldots, \epsilon_{n}^{\prime}}\left(z_{1}, z_{2}, \ldots, z_{n}\right)+\rho_{n,-, \epsilon_{2}, \ldots, \epsilon_{n}}^{-, \epsilon_{2}^{\prime}, \ldots, \epsilon_{n}^{\prime}}\left(z_{1}, z_{2}, \ldots, z_{n}\right)=\rho_{n-1, \epsilon_{2}, \ldots, \epsilon_{n}}^{\epsilon_{2}^{\prime}, \ldots, \epsilon_{n}^{\prime}}\left(z_{2}, \ldots, z_{n}\right) .
$$

- First recurrent relation

$$
\begin{gathered}
\epsilon_{n}^{\prime}, \epsilon_{2}^{\prime}, \ldots, \epsilon_{n}^{\prime} \\
\rho_{n, \epsilon_{1}, \epsilon_{2}, \ldots, \epsilon_{n}}^{\prime}\left(z+1, z, z_{3}, \ldots, z_{n}\right)=-\delta_{\epsilon_{1},-\epsilon_{2}} \epsilon_{1}^{\prime} \epsilon_{2} \rho_{n-1,-\epsilon_{1}^{\prime}, \epsilon_{3}, \ldots, \epsilon_{n}}^{\epsilon_{2}^{\prime}, \epsilon_{3}^{\prime}, \ldots, \epsilon^{\prime}}\left(z, z_{3}, \ldots, z_{n}\right), \\
\epsilon_{1, \epsilon_{2}^{\prime}, \ldots, \epsilon_{n}^{\prime}}^{\prime}\left(z-1, z, z_{3}, \ldots, z_{n}\right)=-\delta_{\epsilon_{1}^{\prime},-\epsilon_{2}^{\prime}} \epsilon_{1} \epsilon_{2}^{\prime} \rho_{n-1, \epsilon_{2}, \epsilon_{3}, \ldots, \epsilon_{n}}^{-\epsilon_{1}, \epsilon_{3}^{\prime}, \ldots, \epsilon_{n}^{\prime}}\left(z, z_{3}, \ldots, z_{n}\right) .
\end{gathered}
$$

- Second recurrent relation

$$
\lim _{z_{1} \rightarrow i \infty} \rho_{n, \epsilon_{1}, \epsilon_{2}, \ldots, \epsilon_{n}}^{\epsilon_{1}^{\prime}, \epsilon_{2}^{\prime}, \ldots, \epsilon_{n}^{\prime}}\left(z_{1}, z_{2}, \ldots, z_{n}\right)=\delta_{\epsilon_{1}, \epsilon_{1}^{\prime}} \frac{1}{2} \rho_{n-1, \epsilon_{2}, \ldots, \epsilon_{n}}^{\epsilon_{2}^{\prime}, \ldots, \epsilon_{n}^{\prime}}\left(z_{2}, \ldots, z_{n}\right) .
$$

- Identity relations

$$
\begin{gathered}
\sum_{\begin{array}{c}
\epsilon_{1}, \ldots, \epsilon_{n} \\
\sum_{i} \epsilon_{i}^{\prime}=\sum_{i} \epsilon_{i}
\end{array}} \rho_{n, \epsilon_{1}, \ldots, \epsilon_{n}}^{\epsilon_{1}^{\prime}, \ldots, \epsilon_{n}^{\prime}}\left(z_{1}, \ldots, z_{n}\right)=\sum_{\substack{\epsilon_{1}^{\prime}, \ldots, \epsilon_{n}^{\prime} \\
\sum_{i} \epsilon_{i}^{\prime}=\sum_{i} \epsilon_{i}}} \rho_{n, \epsilon_{1}, \ldots, \epsilon_{n}}^{\epsilon_{1}^{\prime}, \ldots, \epsilon_{n}^{\prime}}\left(z_{1}, \ldots, z_{n}\right) \\
=\rho_{n,+, \ldots,+}^{+, \ldots,+}\left(z_{1}, \ldots, z_{n}\right)=\rho_{n,-, \ldots,-}^{-, \ldots,-}\left(z_{1}, \ldots, z_{n}\right) .
\end{gathered}
$$

If we assume $\rho_{3}$ as follows

$$
\begin{aligned}
\rho_{3,+++}^{+++}\left(z_{1}, z_{2}, z_{3}\right)= & \frac{1}{8}+A\left(z_{1}, z_{2} \mid z_{3}\right) \omega\left(z_{1}-z_{2}\right)+A\left(z_{1}, z_{3} \mid z_{2}\right) \omega\left(z_{1}-z_{3}\right) \\
& +A\left(z_{2}, z_{3} \mid z_{2}\right) \omega\left(z_{2}-z_{3}\right) \\
\rho_{3,-++}^{-++}\left(z_{1}, z_{2}, z_{3}\right)= & \rho_{2,++}^{++}\left(z_{2}, z_{3}\right)-\rho_{3,+++}^{+++}\left(z_{1}, z_{2}, z_{3}\right), \\
\rho_{3,-++-}^{-+-}\left(z_{1}, z_{2}, z_{3}\right)= & \rho_{2,-+}^{-+}\left(z_{1}, z_{2}\right)-\rho_{3,-++}^{-++}\left(z_{1}, z_{2}, z_{3}\right), \quad \ldots, \\
A\left(z_{1}, z_{2} \mid z_{3}\right)= & \frac{\left(z_{1}-z_{3}\right)\left(z_{2}-z_{3}\right)-1}{12\left(z_{1}-z_{3}\right)\left(z_{2}-z_{3}\right)}
\end{aligned}
$$

these relations are satisfied. In the homogeneous limit $z_{j} \rightarrow 0$ this gives the correct correlation functions of XXX model. In the homogeneous limit each term diverges but we have finite limiting number. Then we can calculate the correlation functions of arbitrary element of density matrix using these algebraic relations, although the calculation become complicated. We have calculated all the inhomogeneous correlation functions up to $n \leq 4$ from the multiple integrals and confirmed these relations are fulfilled.

Further we have found the inhomogeneous correlation functions can be represented in terms of $\omega$-function

$$
\begin{aligned}
& \rho_{n, \epsilon_{1}, \ldots, \epsilon_{n}}^{\epsilon_{1}^{\prime}, \ldots, \epsilon_{n}^{\prime}}\left(z_{1}, \ldots, z_{n}\right)=\left(\prod_{j=1}^{n} \frac{\delta_{\epsilon_{j}, \epsilon_{j}^{\prime}}}{2}\right)+\sum_{m=1}^{\left[\frac{n}{2}\right]} \sum_{1 \leq k_{1}<k_{3}<k_{5}<\cdots<k_{2 m-1}<n, k_{2 m}>k_{2 m-1}}^{\left[\epsilon_{1}\right.} \sum_{A_{\epsilon_{1}, \ldots, \epsilon_{n}}^{\epsilon_{1}^{\prime}, \ldots, \epsilon_{n}^{\prime}}\left(k_{1}, \ldots, k_{2 m} \mid z_{1}, \ldots, z_{n}\right) \omega\left(z_{k_{1}}-z_{k_{2}}\right) \cdots \omega\left(z_{k_{2 m-1}}-z_{k_{2 m}}\right),} \\
& A_{\epsilon_{1}, \ldots, \epsilon_{n}}^{\epsilon_{1}^{\prime}, \ldots, \epsilon_{n}^{\prime}}\left(k_{1}, \ldots, k_{2 m} \mid z_{1}, \ldots, z_{n}\right)=\frac{Q_{\epsilon_{1}, \ldots, \epsilon_{n}}^{\epsilon_{1}^{\prime}, \ldots, \epsilon_{n}^{\prime}}\left(k_{1}, \ldots, k_{2 m} \mid z_{1}, \ldots, z_{n}\right)}{\prod_{i<j}^{\prime}\left(z_{i}-z_{j}\right)} \\
& \quad \text { rational function of } z_{1}, \ldots, z_{n} .
\end{aligned}
$$


Denominator is

$$
\prod_{1 \leq j<k \leq m}\left(z_{k_{2 j-1}}-z_{k_{2 l-1}}\right)\left(z_{k_{2 j-1}}-z_{k_{2 l}}\right)\left(z_{k_{2 j}}-z_{k_{2 l-1}}\right)\left(z_{k_{2 j}}-z_{k_{2 l}}\right) \prod_{l=1}^{2 m}\left(\prod_{i \neq k_{1}, k_{2}, \ldots, k_{2 m}}\left(z_{l}-z_{i}\right)\right) .
$$

The total exponent for this is $4 n m-2 m^{2}-2 m$. The largest exponent for $z_{i}$ is $n-2$ for $i \in\left\{k_{1}, \ldots, k_{2 m}\right\}$ and $2 m$ for $i \neq k_{j}$. Numerator is also polynomials of $z_{1}, \ldots, z_{n}$ which satisfies the same exponent conditions. Unknowns are the coefficients of each terms. Number increases drastically as $n, m$ increases. Algebraic relations give the over complete linear equations. By using mathematica we have unique solution of these equations. By use of algebraic relations, we have calculated all the polynomials for $n=5$

$$
Q_{\epsilon_{1}, \ldots, \epsilon_{5}}^{\epsilon_{1}^{\prime}, \ldots, \epsilon_{5}^{\prime}}\left(k_{1}, k_{2} \mid z_{1}, \ldots, z_{5}\right), \quad Q_{\epsilon_{1}, \ldots, \epsilon_{5}}^{\epsilon_{1}^{\prime}, \ldots, \epsilon_{5}^{\prime}}\left(k_{1}, \ldots, k_{4} \mid z_{1}, \ldots, z_{5}\right)
$$

By the memory problem this calculation stopped at $n=6$.

Fourth-neighbor correlation function [25]

$$
\begin{aligned}
\left\langle S_{j}^{z} S_{j+4}^{z}\right\rangle= & \frac{1}{12}-\frac{16}{3} \ln 2+\frac{145}{6} \zeta(3)-54 \ln 2 \cdot \zeta(3)-\frac{293}{4} \zeta(3)^{2}-\frac{875}{12} \zeta(5)+\frac{1450}{3} \ln 2 \cdot \zeta(5) \\
& -\frac{275}{16} \zeta(3) \cdot \zeta(5)-\frac{1875}{16} \zeta(5)^{2}+\frac{3185}{64} \zeta(7)-\frac{1715}{4} \ln 2 \cdot \zeta(7)+\frac{6615}{32} \zeta(3) \cdot \zeta(7) \\
= & 0.034652776982 \ldots, \\
\left\langle S_{j}^{z} S_{j+5}^{z}\right\rangle= & \frac{1}{12}-\frac{25}{3} \zeta_{a}(1)+\frac{800}{9} \zeta_{a}(3)-\frac{1192}{3} \zeta_{a}(1) \zeta_{a}(3)-\frac{15368}{9} \zeta_{a}(3)^{2}-608 \zeta_{a}(3)^{3} \\
& -\frac{4228}{9} \zeta_{a}(5)+\frac{64256}{9} \zeta_{a}(1) \zeta_{a}(5)-\frac{976}{9} \zeta_{a}(3) \zeta_{a}(5)+3648 \zeta_{a}(1) \zeta_{a}(3) \zeta_{a}(5) \\
& -\frac{3328}{3} \zeta_{a}(3)^{2} \zeta_{a}(5)-\frac{76640}{3} \zeta_{a}(5)^{2}+\frac{66560}{3} \zeta_{a}(1) \zeta_{a}(5)^{2}+\frac{12640}{3} \zeta_{a}(3) \zeta_{a}(5)^{2} \\
& +\frac{6400}{3} \zeta_{a}(5)^{3}+\frac{9674}{9} \zeta_{a}(7)+56952 \zeta_{a}(3) \zeta_{a}(7)-\frac{225848}{9} \zeta_{a}(1) \zeta_{a}(7) \\
& -\frac{116480}{3} \zeta_{a}(1) \zeta_{a}(3) \zeta_{a}(7)-\frac{35392}{3} \zeta_{a}(3)^{2} \zeta_{a}(7)+7840 \zeta_{a}(5) \zeta_{a}(7) \\
& -8960 \zeta_{a}(3) \zeta_{a}(5) \zeta_{a}(7)-\frac{66640}{3} \zeta_{a}(7)^{2}+31360 \zeta_{a}(1) \zeta_{a}(7)^{2}-686 \zeta_{a}(9) \\
& +18368 \zeta_{a}(1) \zeta_{a}(9)-53312 \zeta_{a}(3) \zeta_{a}(9)+35392 \zeta_{a}(1) \zeta_{a}(3) \zeta_{a}(9) \\
& +16128 \zeta_{a}(3)^{2} \zeta_{a}(9)+38080 \zeta_{a}(5) \zeta_{a}(9)-53760 \zeta_{a}(1) \zeta_{a}(5) \zeta_{a}(9) \\
= & -0.03089036664760932 \ldots
\end{aligned}
$$

Using this algebraic method we can calculate all the element of density sub-matrix of successive 6 -sites. Longer system is quite difficult because of the memory and computing time problem. We can calculate 6 -th neighbor and 7-th neighbor correlations using the generation function method. They are represented by long polynomials of $\zeta_{a}$ 's. Here we write only numerical results [26]

$$
\left\langle S_{j}^{z} S_{j+6}^{z}\right\rangle=0.02444673832795890 \ldots, \quad\left\langle S_{j}^{z} S_{j+7}^{z}\right\rangle=-0.0224982227633722 \ldots
$$

\subsection{Calculation by continuous dimensions}

In a series of papers Boos, Jimbo, Miwa, Smirnov and Takeyama formulated these algebraic calculation by the trace of continuous dimension of auxiliary space $[27,28,29,30]$

$$
\left(\rho_{n}\right)_{\epsilon_{1}, \ldots, \epsilon_{n}}^{\bar{\epsilon}_{1}, \ldots, \bar{\epsilon}_{n}}=\left\langle\operatorname{vac}\left|\left(E_{\epsilon_{1}}^{\bar{\epsilon}_{1}}\right)_{1} \cdots\left(E_{\epsilon_{n}}^{\bar{\epsilon}_{n}}\right)_{n}\right| \operatorname{vac}\right\rangle
$$




$$
\begin{aligned}
& h_{n}\left(\epsilon_{1}, \ldots, \epsilon_{n}, \bar{\epsilon}_{n}, \ldots, \bar{\epsilon}_{1}\right)=(-1)^{n}\left(\prod_{j=1}^{n} \bar{\epsilon}_{j}\right)\left(\rho_{n}\right)_{\epsilon_{1}, \ldots, \epsilon_{n}}^{-\bar{\epsilon}_{1}, \ldots,-\bar{\epsilon}_{n}}, \\
& s_{n}=\prod_{j=1}^{n} \frac{1}{2}\left(|+\rangle_{j}|-\rangle_{\bar{j}}-|-\rangle_{j}|+\rangle_{\bar{j}}\right), \quad h_{n}=\exp \left(\Omega_{n}\right) s_{n} .
\end{aligned}
$$

Density sub-matrix in $2^{n}$ dimensional space is mapped to a vector in $2^{2 n}$ dimensional space. $\Omega_{n}$ is an operator in this space. Monodoromy matrix is defined as follows:

$$
\begin{aligned}
& L_{j}^{(0)}(\lambda)=\left(\lambda+\frac{1}{2}\right) I \sigma_{j}^{0}+\frac{1}{2}\left(H \sigma_{j}^{z}+2 E \sigma_{j}^{+}+2 F \sigma_{j}^{-}\right), \\
& T_{n}(\lambda)=L_{\overline{1}}^{(0)}\left(\lambda-z_{1}-1\right) \cdots L_{\bar{n}}^{(0)}\left(\lambda-z_{n}-1\right) L_{n}^{(0)}\left(\lambda-z_{n}\right) \cdots L_{1}^{(0)}\left(\lambda-z_{1}\right) .
\end{aligned}
$$

Here $I, H, E, F$ are $d \times d$ matrices

$$
I_{i, j}=\delta_{i, j}, \quad H_{i, j}=(d+1-2 i) \delta_{i, j}, \quad E_{i, j}=(i-1) \delta_{i, j+1}, \quad F_{i, j}=(d-i) \delta_{i+1, j} .
$$

These satisfy commutation relations $[H, E]=-2 E,[H, F]=2 F,[E, F]=-H . \sigma_{j}^{z}, \sigma_{j}^{+}, \sigma_{j}^{-}$are Pauli operators in $2 n$ spin space $j=1, \ldots, n, \overline{1}, \ldots, \bar{n}$. Especially at $d=2$ we have

$$
I=\left(\begin{array}{ll}
1 & 0 \\
0 & 1
\end{array}\right), \quad H=\left(\begin{array}{cc}
1 & 0 \\
0 & -1
\end{array}\right), \quad E=\left(\begin{array}{ll}
0 & 0 \\
1 & 0
\end{array}\right), \quad F=\left(\begin{array}{ll}
0 & 1 \\
0 & 0
\end{array}\right),
$$

and

$$
T_{n}(\lambda)=\left(\begin{array}{ll}
A(\lambda) & B(\lambda) \\
C(\lambda) & D(\lambda)
\end{array}\right)
$$

The trace of any monomial of $I, E, H$ and $F$ is a polynomial of dimension $d$. One can calculate from the definition (5). For example,

$$
\operatorname{Tr}_{d} I=d, \quad \operatorname{Tr}_{d} H H=\left(d^{3}-d\right) / 3, \quad \operatorname{Tr}_{d} H=0 .
$$

The dimension $d$ is replaced by $\mu-\nu$. Following [30], the operator $\Omega_{n}$ is given by

$$
\begin{aligned}
\Omega_{n}= & \frac{1}{2} \oint \frac{d \mu}{2 \pi i} \frac{d \nu}{2 \pi i} \frac{(\mu-\nu) \omega(\mu-\nu)}{\left(1-(\mu-\nu)^{2}\right)^{2} \prod_{j=1}^{n}\left(\mu-z_{j}\right)\left(1-\left(\mu-z_{j}\right)^{2}\right)\left(\nu-z_{j}\right)\left(1-\left(\nu-z_{j}\right)^{2}\right)} \\
& \times \operatorname{Tr}_{\mu-\nu} T_{n}\left(\frac{\mu+\nu}{2}\right)(A(\mu) D(\nu)+D(\mu) A(\nu)-B(\mu) C(\nu)-C(\mu) B(\nu)),
\end{aligned}
$$

where the integration path should surround all $z_{j}$ counter-clockwise. In the homogeneous limit $z_{j} \rightarrow 0 \Omega$ becomes

$$
\begin{aligned}
\Omega_{n}= & \frac{1}{2} \oint \frac{d \mu}{2 \pi i} \frac{d \nu}{2 \pi i} \frac{(\mu-\nu) \omega(\mu-\nu)}{\left(1-(\mu-\nu)^{2}\right)^{2} \mu^{n}\left(1-\mu^{2}\right)^{n} \nu^{n}\left(1-\nu^{2}\right)^{n}} \\
& \times \operatorname{Tr}_{\mu-\nu} T_{n}\left(\frac{\mu+\nu}{2}\right)(A(\mu) D(\nu)+D(\mu) A(\nu)-B(\mu) C(\nu)-C(\mu) B(\nu)),
\end{aligned}
$$

and the calculation becomes very simple. By this formulation we could calculate all the elements of density sub-matrix at $n=7$. Calculating the eigenvalues of matrix, we can calculate the von Neumann entropy (entanglement entropy) up to seven sites,

$$
S(n) \equiv-\operatorname{tr} \rho_{n} \log _{2} \rho_{n}=-\sum_{\alpha=1}^{2^{n}} \omega_{\alpha} \log _{2} \omega_{\alpha},
$$

where $\omega_{\alpha}$ are eigenvalues of density sub-matrix $\rho_{n}$. In Table $1 S(n)$ is given up to $n=7$. 
Table 1. von Neumann entropy $S(n)$ of a finite sub-chain of length $n$.

\begin{tabular}{cccc}
\hline$S(1)$ & $S(2)$ & $S(3)$ & $S(4)$ \\
\hline 1 & 1.3758573262887466 & 1.5824933209573855 & 1.7247050949099274 \\
\hline & \multicolumn{3}{|c}{} \\
\cline { 2 - 4 } & $S(5)$ & $S(6)$ & $S(7)$ \\
\cline { 2 - 4 } & 1.833704916848315 & 1.922358833819333 & 1.997129812895912 \\
\hline
\end{tabular}

\subsection{Generalization to XXZ model}

In 2003, Kato, Shiroishi, Takahashi, Sakai have generalized the Boos-Korepin method to the XXZ models with an anisotropy parameter $|\Delta| \leq 1$ for successive three sites [31]. For example $P(n)$ is represented as follows:

$$
\begin{aligned}
P(n)= & (-\nu)^{-\frac{n(n-1)}{2}} \int_{-\infty}^{\infty} \frac{\mathrm{d} x_{1}}{2 \pi} \cdots \int_{-\infty}^{\infty} \frac{\mathrm{d} x_{n}}{2 \pi} \prod_{a>b} \frac{\sinh \left(x_{a}-x_{b}\right)}{\sinh \left(\left(x_{a}-x_{b}-\mathrm{i} \pi\right) \nu\right)} \\
& \times \prod_{j=1}^{n} \frac{\sinh ^{n-j}\left(\left(x_{j}+\frac{\mathrm{i} \pi}{2}\right) \nu\right) \sinh ^{j-1}\left(\left(x_{j}-\frac{\mathrm{i} \pi}{2}\right) \nu\right)}{\cosh ^{n} x} .
\end{aligned}
$$

Here $\Delta=\cos (\pi \nu)$. Similar integral representations for any arbitrary correlation function for successive three sites were calculated.

Nearest-neighbor correlation functions

$$
\left\langle S_{j}^{x} S_{j+1}^{x}\right\rangle=\frac{1}{4 \pi s_{1}} \zeta_{\nu}(1)+\frac{c_{1}}{4 \pi^{2}} \zeta_{\nu}^{\prime}(1), \quad\left\langle S_{j}^{z} S_{j+1}^{z}\right\rangle=\frac{1}{4}-\frac{c_{1}}{2 \pi s_{1}} \zeta_{\nu}(1)-\frac{1}{2 \pi^{2}} \zeta_{\nu}^{\prime}(1) .
$$

Next nearest-neighbor correlation functions

$$
\begin{aligned}
\left\langle S_{j}^{x} S_{j+2}^{x}\right\rangle & =\frac{1}{2 \pi s_{2}} \zeta_{\nu}(1)+\frac{c_{2}}{4 \pi^{2}} \zeta_{\nu}^{\prime}(1)-\frac{3\left(1-c_{2}\right) c_{2}}{8 \pi s_{2}} \zeta_{\nu}(3)-\frac{s_{1}^{2}}{8 \pi^{2}} \zeta_{\nu}^{\prime}(3), \\
\left\langle S_{j}^{z} S_{j+2}^{z}\right\rangle & =\frac{1}{4}-\frac{1+2 c_{2}}{\pi s_{2}} \zeta_{\nu}(1)-\frac{1}{2 \pi^{2}} \zeta_{\nu}^{\prime}(1)+\frac{3 s_{1}}{4 \pi c_{1}} \zeta_{\nu}(3)+\frac{1-c_{2}}{8 \pi^{2}} \zeta_{\nu}^{\prime}(3) .
\end{aligned}
$$

Here

$$
\begin{aligned}
& c_{j}:=\cos \pi j \nu, \quad s_{j}:=\sin \pi j \nu, \\
& \zeta_{\nu}(j):=\int_{-\infty-\frac{\pi i}{2}}^{\infty-\frac{\pi i}{2}} \mathrm{~d} x \frac{1}{\sinh x} \frac{\cosh \nu x}{\sinh ^{j} \nu x}, \quad \zeta_{\nu}^{\prime}(j):=\int_{-\infty-\frac{\pi i}{2}}^{\infty-\frac{\pi i}{2}} \mathrm{~d} x \frac{1}{\sinh x} \frac{\partial}{\partial \nu} \frac{\cosh \nu x}{\sinh ^{j} \nu x} .
\end{aligned}
$$

Replacing $\nu \rightarrow i \eta / \pi$, we can also get the correlation functions in the massive region $\Delta=\cosh \eta>$ 1 [32]. Third neighbor correlations is also expressed by functions $\zeta_{\nu}$ and $\zeta_{\nu}^{\prime}$, although the expression becomes more complicated [33]. In Figs. 1 and 2, the nearest neighbor, the second neighbor and the third neighbor correlations are shown as functions of $\Delta$.

\section{Simplified thermodynamic Bethe ansatz equation}

Simplified thermodynamic Bethe ansatz equation at temperature $T[9,10]$ is

$$
\begin{aligned}
u(x) & -2 \cosh (h / T)-\oint \frac{\theta}{2}\left[\operatorname{coth} \frac{\theta}{2}(x-y-2 i) \exp \left(-\frac{2 \pi J \sin \theta}{\theta T} a_{1}(y+i)\right)\right. \\
& \left.+\operatorname{coth} \frac{\theta}{2}(x-y+2 i) \exp \left(-\frac{2 \pi J \sin \theta}{\theta T} a_{1}(y-i)\right)\right] \frac{1}{u(y)} \frac{d y}{2 \pi i}=0,
\end{aligned}
$$




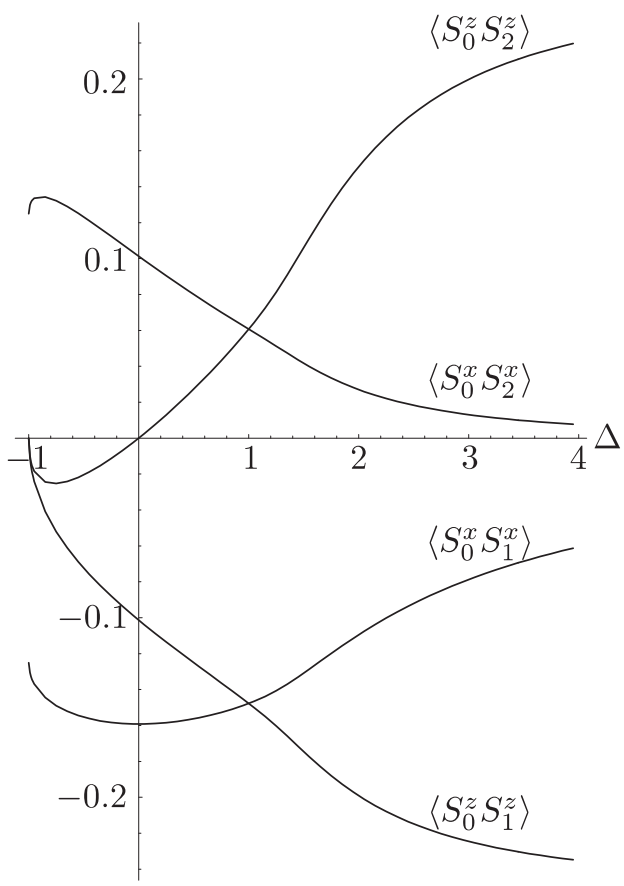

Figure 1. The nearest-neighbor and the next nearest neighbor correlation functions for the XXZ chain. We calculated $\left\langle S_{j}^{z} S_{j+1}^{z}\right\rangle,\left\langle S_{j}^{x} S_{j+1}^{x}\right\rangle,\left\langle S_{j}^{z} S_{j+2}^{z}\right\rangle$ and $\left\langle S_{j}^{x} S_{j+2}^{x}\right\rangle$.

where

$$
a_{1}(x) \equiv \frac{\theta \sin \theta}{2 \pi(\cosh \theta x-\cos \theta)} .
$$

Free energy per site is

$$
f=-T \ln u(0) .
$$

In this section we look for analytic solution for XY case $\Delta=0, \theta=\pi / 2$. For this case equation (7) becomes

$$
u(x)-2 \cosh (h / T)-\oint \frac{\pi}{4} \tanh \frac{\pi}{4}(x-y) 2 \cos \left(\frac{J}{T \sinh \pi y / 2}\right) \frac{1}{u(y)} \frac{d y}{2 \pi i}=0 .
$$

Putting $X=\tanh \pi x / 4, Y=\tanh \pi y / 4$ and using $u(-Y)=u(Y)$ we have

$$
u(X)-2 \cosh (h / T)+2\left(1-X^{2}\right) \oint \cos \left(\frac{J}{2 T} \frac{1-Y^{2}}{Y}\right) \frac{Y}{\left(1-X^{2} Y^{2}\right)\left(1-Y^{2}\right) u(Y)} \frac{d Y}{2 \pi i}=0 .
$$

Consider the Fourier transform of following function

$$
g(\theta)=\frac{1}{2} \ln \left(4\left(\cosh ^{2}\left(\frac{J}{2 T} \sin \theta\right)+\sinh ^{2}(h / T)\right)\right), \quad a_{j} \equiv \int_{0}^{2 \pi} g(\theta) \cos (2 j \theta) \frac{d \theta}{2 \pi} .
$$

Assume that

$$
\ln u(X)=a_{0}+2 \sum_{j=1}^{\infty} a_{j} X^{2 j} .
$$




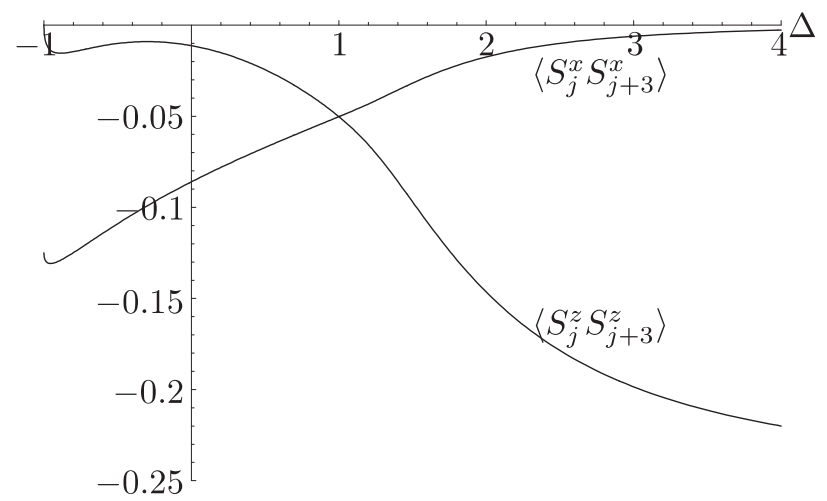

Figure 2. The third-neighbor correlation functions for the XXZ chain.

We can show that this satisfies (8). This series is convergent at $|X| \leq 1$.

$$
\begin{aligned}
& \ln u\left(e^{i \theta}\right)+\ln u\left(e^{-i \theta}\right)=2 a_{0}+2 \sum_{j=1}^{\infty} a_{j} \cos (2 j \theta) \\
& =2 g(\theta)=\ln \left(4\left(\cosh ^{2}\left(\frac{J}{2 T} \sin \theta\right)+\sinh ^{2}(h / T)\right)\right) .
\end{aligned}
$$

Then we have

$$
u(X) u(1 / X)=2 \cos \left(\frac{J}{2 T}(X-1 / X)\right)+2+4 \sinh ^{2}(h / T) .
$$

The function $u(X)$ has zeros at $X= \pm \beta_{j, \pm}$, where $\beta_{j, \pm}=\alpha_{j, \pm}+\sqrt{1+\alpha_{j, \pm}^{2}}$, and $\alpha_{j, \pm}=$ $\frac{2 \pi T}{J}\left(j-\frac{1}{2}\right) \pm \frac{2 h i}{J}$. One can show that $\left|\beta_{j, \pm}\right|>1$. $u(X)$ should not have zeros at $|X|<1$. Then we have $u(X)=$ const $\cdot \prod_{j=1}^{\infty}\left(1-\frac{X^{2}}{\beta_{j,+}^{2}}\right)\left(1-\frac{X^{2}}{\beta_{j,-}^{2}}\right)$. By the condition $u(1)=2 \cosh (h / T)$ we have infinite product expansion of $u(X)$

$$
u(X)=2 \cosh (h / T) \prod_{j=1}^{\infty} \frac{\left(1-\frac{X^{2}}{\beta_{j,+}^{2}}\right)\left(1-\frac{X^{2}}{\beta_{j,-}^{2}}\right)}{\left(1-\frac{1}{\beta_{j,+}^{2}}\right)\left(1-\frac{1}{\beta_{j,-}^{2}}\right)} .
$$

From (10) we have

$$
2 \cos \left(\frac{J}{2 T}(Y-1 / Y)\right) / u(Y)=u(1 / Y)-\left(2+4 \sinh ^{2}(h / T)\right) / u(Y)
$$

Then we can write $u(X)=2 \cosh (h / T)+v\left(X^{2}\right)\left(1-X^{2}\right), v(X)=\sum_{j=0}^{\infty} d_{j} X^{j}$. Convergence radius of $v(X)$ is infinite

$$
\begin{aligned}
\text { l.h.s. of }(8) & =u(X)-2 \cosh (h / T)+\left(1-X^{2}\right) \oint u(1 / Y) \frac{Y}{\left(1-X^{2} Y^{2}\right)\left(1-Y^{2}\right)} \frac{d Y}{\pi i} \\
& =\left(1-X^{2}\right)\left[v\left(X^{2}\right)-\oint v\left(1 / Y^{2}\right) \frac{1}{Y\left(1-X^{2} Y^{2}\right)} \frac{d Y}{2 \pi i}\right] \\
& =\left(1-X^{2}\right) \sum_{j=0}^{\infty} d_{j}\left(X^{2 j}-\oint \frac{1}{Y^{2 j+1}\left(1-X^{2} Y^{2}\right)} \frac{d Y}{2 \pi i}\right)=0 .
\end{aligned}
$$


Thus we have proved that (11), (9) satisfies the equation (8). The free energy

$$
-T \ln u(0)=-T a_{0}
$$

coincides with the known result [34].

\section{Summary}

For $J<0, \Delta=1, T=h=0$ we obtained the factorized form of density sub-matrix up to $n=7$. The entanglement entropy for seven sites is new result of this paper. Up to six sites we published in [26].

The six-th neighbor and the seven-th neighbor correlations are calculated by the generating function method for $J<0, \Delta=1, T=h=0$ [26].

For arbitrary $\Delta, T=h=0$ we obtained the factorized form up to $n=4$. Correlations are given by two transcendental functions $\zeta_{\nu}(j)$ and $\zeta_{\nu}^{\prime}(j)$ with $j=1,3,5, \ldots$ defined by (6). For correlations of $n \geq 5$ the calculation becomes very tedious and no one has succeeded.

For simplified TBA equation, we obtained the analytic solution for XY limit $\Delta=0$. Analytic solution for Ising limit was given in [9].

\section{A Strong coupling expansion of the Hubbard model}

The Hubbard Hamiltonian is written as follows:

$$
\mathcal{H}=-t \sum_{<i j>} \sum_{\sigma}\left(c_{i \sigma}^{\dagger} c_{j \sigma}+c_{j \sigma}^{\dagger} c_{i \sigma}\right)+U \sum_{i=1}^{N_{a}} c_{i \uparrow}^{\dagger} c_{i \uparrow} c_{i \downarrow}^{\dagger} c_{i \downarrow} .
$$

If we treat the interaction term as main Hamiltonian and hopping therm as perturbation in the half-filled case

$$
\begin{aligned}
& \mathcal{H}_{0}=U \sum n_{i \uparrow} n_{i \downarrow}, \\
& \mathcal{H}_{1}=-\sum_{\sigma} \sum_{i<j} t_{i, j}\left(c_{i \sigma}^{\dagger} c_{j \sigma}+c_{j \sigma}^{\dagger} c_{i \sigma}\right)
\end{aligned}
$$

the effective Hamiltonian becomes as follows:

$$
\begin{aligned}
\mathcal{H}_{\mathrm{eff}}= & \sum_{i<j} \frac{t_{i j} t_{j i}}{U}\left(\sigma_{i} \cdot \sigma_{j}-1\right)+U^{-3}\left[\sum_{i<j} t_{i j}^{4}\left(1-\sigma_{i} \cdot \sigma_{j}\right)+\sum_{i<k} t_{i j}^{2} t_{j k}^{2}\left(\sigma_{i} \cdot \sigma_{k}-1\right)\right. \\
& +\sum_{i<j<l, i<k, k \neq j, l} t_{i j} t_{j k} t_{k l} t_{l i}\left(5\left(\sigma_{j} \cdot \sigma_{k}\right)\left(\sigma_{i} \cdot \sigma_{l}\right)+5\left(\sigma_{i} \cdot \sigma_{j}\right)\left(\sigma_{k} \cdot \sigma_{l}\right)-5\left(\sigma_{j} \cdot \sigma_{k}\right)\left(\sigma_{i} \cdot \sigma_{l}\right)\right. \\
& \left.\left.-\sigma_{i} \cdot \sigma_{j}-\sigma_{j} \cdot \sigma_{k}-\sigma_{k} \cdot \sigma_{l}-\sigma_{l} \cdot \sigma_{i}-\sigma_{i} \cdot \sigma_{k}-\sigma_{j} \cdot \sigma_{l}+1\right)\right] \\
\sigma_{i} \cdot \sigma_{j} & =4 \mathbf{S}_{i} \cdot \mathbf{S}_{j} .
\end{aligned}
$$

For one-dimensional half-filled case the four spin term disappears and the effective Hamiltonian becomes

$$
\frac{t^{2}}{U} \sum_{i}\left(4 \mathbf{S}_{i} \cdot \mathbf{S}_{i+1}-1\right)+\frac{t^{4}}{U^{3}} \sum_{i}\left\{4\left(1-4 \mathbf{S}_{i} \cdot \mathbf{S}_{i+1}\right)+\left(4 \mathbf{S}_{i} \cdot \mathbf{S}_{i+2}-1\right)\right\}+O\left(\frac{t^{6}}{U^{5}}\right) .
$$


On the other hand exact ground state energy per site is expanded as [35, 36]

$$
\begin{aligned}
e & =-4|t| \int_{0}^{\infty} \frac{J_{0}(\omega) J_{1}(\omega) d \omega}{\omega\left[1+\exp \left(2 U^{\prime} \omega\right)\right]} \\
& =-4|t|\left[\left(\frac{1}{2}\right)^{2} \ln 2 U^{\prime}-1-\left(\frac{1 \cdot 3}{2 \cdot 4}\right)^{2} \frac{\zeta(3)}{3}\left(1-\frac{1}{2^{2}}\right) U^{\prime}-3+\cdots\right] \\
U^{\prime} & \equiv U /(4|t|) .
\end{aligned}
$$

Comparing the first term of (12) and (13), we get nearest neighbor correlation (2). From the second term we get the second neighbor correlation (3).

\section{B Transformation to canonical form in case of $P(3)$}

$$
T_{3}=\frac{\left(\lambda_{1}+i\right)^{2}\left(\lambda_{2}+i\right) \lambda_{2} \lambda_{3}^{2}}{\left(\lambda_{3}-\lambda_{1}-i\right)\left(\lambda_{3}-\lambda_{2}-i\right)\left(\lambda_{2}-\lambda_{1}-i\right)} .
$$

is decomposed to the following three terms,

$$
=\frac{i\left(\lambda_{1}+i\right)^{2}\left(\lambda_{2}+i\right) \lambda_{2} \lambda_{3}^{2}}{\left(\lambda_{3}-\lambda_{1}-i\right)\left(\lambda_{2}-\lambda_{1}-i\right)}+\frac{i\left(\lambda_{1}+i\right)^{2}\left(\lambda_{2}+i\right) \lambda_{2} \lambda_{3}^{2}}{\left(\lambda_{3}-\lambda_{1}-i\right)\left(\lambda_{3}-\lambda_{2}-i\right)}-\frac{i\left(\lambda_{1}+i\right)^{2}\left(\lambda_{2}+i\right) \lambda_{2} \lambda_{3}^{2}}{\left(\lambda_{3}-\lambda_{2}-i\right)\left(\lambda_{2}-\lambda_{1}-i\right)} .
$$

Using the antisymmetry of $U_{3}\left(\lambda_{1}, \lambda_{2}, \lambda_{3}\right)$ the first and the second terms are simplified as follows:

the first term $\sim \lambda_{1}^{2} \lambda_{2}-\frac{\left(\lambda_{1}+i\right)^{3} \lambda_{3}}{\lambda_{2}-\lambda_{1}-i}$

the second term $\sim \lambda_{1}^{2} \lambda_{2}-\frac{\left(\lambda_{1}+i\right)^{3}\left(\lambda_{3}+i\right)}{\lambda_{2}-\lambda_{1}-i}$.

The third term is transformed as follows

the third term $\sim-\lambda_{1}^{2} \lambda_{2}-i \frac{\left(\lambda_{1}+i\right)^{3}\left(\lambda_{3}+i\right)^{2}}{\lambda_{2}-\lambda_{1}-i}+i \frac{\left(\lambda_{1}+i\right)^{3} \lambda_{3}^{2}}{\lambda_{2}-\lambda_{1}-i}-\frac{i\left(\lambda_{1}+i\right)^{3} \lambda_{3}^{3}}{\left(\lambda_{3}-\lambda_{2}-i\right)\left(\lambda_{2}-\lambda_{1}-i\right)}$.

Then $T_{3}$ is transformed as follows:

$$
T_{3} \sim-\lambda_{2} \lambda_{3}^{2}-\frac{i\left(\lambda_{1}+i\right)^{3} \lambda_{3}^{3}}{\left(\lambda_{3}-\lambda_{2}-i\right)\left(\lambda_{2}-\lambda_{1}-i\right)} .
$$

We should note that the pole at $\lambda_{3}=0$ and $\lambda_{1}=-i$ of $U_{3}$ is canceled by numerator of the second term. So we can change the integration path $\lambda_{1} \rightarrow \lambda_{1}-i$ and $\lambda_{3} \rightarrow \lambda_{3}+i$

$$
T_{3} \sim-\lambda_{2} \lambda_{3}^{2}-\frac{i \lambda_{1}^{3}\left(\lambda_{3}+i\right)^{3}}{\left(\lambda_{3}-\lambda_{2}\right)\left(\lambda_{2}-\lambda_{1}\right)} .
$$

Using the antisymmetry of $U$ we have

$$
\sim-\lambda_{2} \lambda_{3}^{2}-\frac{3 \lambda_{1}^{2} \lambda_{3}^{2}+3 i \lambda_{1} \lambda_{3}^{2}+3 i \lambda_{1}^{2} \lambda_{3}-\lambda_{3}^{2}-\lambda_{3} \lambda_{1}-\lambda_{1}^{2}}{\lambda_{2}-\lambda_{1}} .
$$

Thus the denominator is drastically simplified. Using the following relations

$$
\frac{\lambda_{1}^{2}}{\lambda_{2}-\lambda_{1}} f\left(\lambda_{3}\right) \sim\left(\frac{-i \lambda_{1}+\frac{1}{3}}{\lambda_{2}-\lambda_{1}}-\frac{1}{3}\left(\lambda_{1}+i\right)\right) f\left(\lambda_{3}\right),
$$


we can reduce the power of numerator,

$$
T_{3} \sim-2 \lambda_{2} \lambda_{3}^{2}+\frac{\frac{1}{3}-i \lambda_{1}-i \lambda_{3}-2 \lambda_{1} \lambda_{3}}{\lambda_{2}-\lambda_{1}}
$$

Thus we have obtained the canonical form for $P(3)$. The derivation of (14) is as follows:

$$
\begin{aligned}
& \frac{\lambda_{1}^{3}}{\lambda_{2}-\lambda_{1}} f\left(\lambda_{3}\right) \sim-\frac{\left(\lambda_{1}+i\right)^{3}}{\lambda_{2}-\lambda_{1}-i} f\left(\lambda_{3}\right)=\left(\frac{\lambda_{2}^{3}-\left(\lambda_{1}+i\right)^{3}}{\lambda_{2}-\lambda_{1}-i}-\frac{\lambda_{2}^{3}}{\lambda_{2}-\lambda_{1}-i}\right) f\left(\lambda_{3}\right) \\
& \sim\left(\frac{\lambda_{2}^{3}-\left(\lambda_{1}+i\right)^{3}}{\lambda_{2}-\lambda_{1}-i}+\frac{\left(\lambda_{2}+i\right)^{3}}{\lambda_{2}-\lambda_{1}}\right) f\left(\lambda_{3}\right) \sim\left(\frac{\lambda_{2}^{3}-\left(\lambda_{1}+i\right)^{3}}{\lambda_{2}-\lambda_{1}-i}+\frac{\left(\lambda_{1}+i\right)^{3}}{\lambda_{2}-\lambda_{1}}\right) f\left(\lambda_{3}\right) .
\end{aligned}
$$

\section{Acknowledgements}

The author acknowledges to A. Klümper, F. Göhmann, H. Boos, J. Sato and M. Shiroishi for stimulating discussions. This work is financially supported by DFG.

\section{References}

[1] Takahashi M., Half-filled Hubbard model at low temperature, J. Phys. C 10 (1977), 1289-1301.

[2] Yang C.N., Yang C.P., Thermodynamics of a one-dimensional system of bosons with repulsive delta-function interaction, J. Math. Phys. 10 (1969), 1115-1122.

[3] Takahashi M., One-dimensional Heisenberg model at finite temperature, Prog. Theor. Phys. 46 (1971), 401-415.

[4] Gaudin M., Thermodynamics of the Heisenberg-Ising ring for $\Delta \geq 1$, Phys. Rev. Lett. 26 (1971), 1301-1304.

[5] Takahashi M., Suzuki M., One-dimensional anisotropic Heisenberg model at finite temperatures, Prog. Theor. Phys. 46 (1972), 2187-2209.

[6] Koma T., Thermal Bethe-ansatz method for the spin-1/2 XXZ Heisenberg chain, Prog. Theor. Phys. 81 (1989), 783-809.

[7] Takahashi M., Correlation length and free energy of $S=1 / 2$ XXZ chain in magnetic field, Phys. Rev. B 44 (1991), 12382-12394.

[8] Klümper A., Thermodynamics of the anisotropic spin-1/2 Heisenberg chain and related quantum chains, Z. Phys. B 91 (1993), 507-519, cond-mat/9306019.

[9] Takahashi M., Simplification of thermodynamic Bethe-ansatz equations, in Physics and Combinatrix (Nagoya, 2000), World Sci. Publ., River Edge, NJ, 2001, 299-304, cond-mat/0010486.

[10] Takahashi M., Shiroishi M., Klümper A., Equivalence of TBA and QTM, J. Phys. A: Math. Gen. 34 (2001), L187-L194, cond-mat/0102027.

[11] Shiroishi M., Takahashi M., Integral equation generates high-temperature expansion of the Heisenberg chain, Phys. Rev. Lett. 89 (2002), 117201, 4 pages, cond-mat/0205180.

[12] Tsuboi Z., Takahashi M., Nonlinear integral equations for thermodynamics of the $U_{q}(\widehat{s l(r+1)})$ Perk-Schultz model, J. Phys. Soc. Japan 74 (2005), 898-904, cond-mat/0412698.

[13] Hulthén L., Über das Austauschproblem eines Kristalles, Ark. Mat. Astron. Fys. A 26 (1938), 1-105.

[14] Muramoto N., Takahashi M., Integrable magnetic model of two chains coupled by four-body interactions, J. Phys. Soc. Japan 68 (1999), 2098-2104, cond-mat/9902007.

[15] Jimbo M., Miki K., Miwa T., Nakayashiki A., Correlation functions of the XXZ model for $\Delta<-1$, Phys. Lett. A 168 (1992), 256-263, hep-th/9205055.

[16] Nakayashiki A., Some integral formulas for the solutions of the $s l_{2}$ dKZ equation with level-4, Internat. J. Modern Phys. A 9 (1994), 5673-5687.

[17] Jimbo M., Miwa T., Quantum KZ equation with $|q|=1$ and correlation functions of the XXZ model in the gapless regime, J. Phys. A: Math. Gen. 29 (1996), 2923-2958, hep-th/9601135.

[18] Kitanine N., Maillet J.M., Terras V., Correlation functions of the XXZ Heisenberg spin-1/2 chain in a magnetic field, Nuclear Phys. B 567 (2000), 554-582, math-ph/9907019. 
[19] Göhmann F., Klümper A., Seel A., Integral representations for correlation functions of the XXZ chain at finite temperature, J. Phys. A: Math. Gen. 37 (2004), 7625-7651, hep-th/0405089.

[20] Boos H.E., Korepin V.E., Quantum spin chains and Riemann zeta function with odd arguments, J. Phys. A: Math. Gen. 34 (2001), 5311-5316, hep-th/0104008.

[21] Boos H.E., Korepin V.E., Evaluation of integrals representing correlations in XXX Heisenberg spin chain, in MathPhys Odyssey (2001), Prog. Math. Phys., Vol. 23, Birkhäuser Boston, Boston, MA, 2002, 65-108, hep-th/0105144.

[22] Sakai K., Shiroishi M., Nishiyama Y., Takahashi M., Third-neighbor correlators of a one-dimensional spin1/2 Heisenberg antiferromagnet, Phys. Rev. E 67 (2003), 065101(R), 4 pages, cond-mat/0302564.

[23] Boos H.E., Korepin V.E., Nishiyama Y., Shiroishi M., Quantum correlations and number theory, J. Phys. A: Math. Gen. 35 (2002), 4443-4451, cond-mat/0202346.

[24] Boos H.E., Korepin V.E., Smirnov F.A., Emptiness formation probability and quantum KnizhnikZamolodchikov equation, Nuclear Phys. B 658 (2003), 417-439, hep-th/0209246.

[25] Boos H.E., Shiroishi M., Takahashi M., First principle approach to correlation functions of spin-1/2 Heisenberg chain: fourth-neighbor correlators, Nuclear Phys. B 712 (2005), 573-599, hep-th/0410039.

[26] Sato J., Shiroishi M., Takahashi M., Correlation functions of the spin-1/2 anti-ferromagnetic Heisenberg chain: exact calculation via the generating function, Nuclear Phys. B 729 (2005), 441-466, hep-th/0507290.

[27] Boos H., Jimbo M., Miwa T., Smirnov F., Takeyama Y., A recursion formula for the correlation functions of an inhomogeneous XXX model, St. Petersburg Math. J. 17 (2005), 85-117, hep-th/0405044.

[28] Boos H., Jimbo M., Miwa T., Smirnov F., Takeyama Y., Reduced qKZ equation and correlation functions of the XXZ model, Comm. Math. Phys. 261 (2006), 245-276, hep-th/0412191.

[29] Boos H., Jimbo M., Miwa T., Smirnov F., Takeyama Y., Traces on the Sklyanin algebra and correlation functions of the eight-vertex model, J. Phys. A: Math. Gen. 38 (2005), 7629-7659, hep-th/0504072.

[30] Boos H., Jimbo M., Miwa T., Smirnov F., Takeyama Y., Density matrix of a finite sub-chain of the Heisenberg anti-ferromagnet, Lett. Math. Phys. 75 (2006), 201-208, hep-th/0506171.

[31] Kato G., Shiroishi M., Takahashi M., Sakai K., Next nearest-neighbor correlation functions of the spin-1/2 XXZ chain at critical region, J. Phys. A: Math. Gen. 36 (2003), L337-L344, cond-mat/0304475.

[32] Takahashi M., Kato G., Shiroishi M., Next nearest-neighbor correlation functions of the spin-1/2 XXZ chain at massive region, J. Phys. Soc. Japan 73 (2004), 245-253, cond-mat/0308589.

[33] Kato G., Shiroishi M., Takahashi M. Sakai K., Third-neighbour and other four-point correlation functions of spin-1/2 XXZ chain, J. Phys. A: Math. Gen. 37 (2004), 5097-5123, cond-mat/0402625.

[34] Takahashi M., Thermodynamics of one-dimensional solvable models, Cambridge University Press, Cambridge, 1999.

[35] Lieb E.H., Wu F.Y., Absence of mott transition in an exact solution of the short-range, one-band model in one dimension, Phys. Rev. Lett. 20 (1968), 1445-1448, Erratum, Phys. Rev. Lett. 21 (1968), 192.

[36] Takahashi M., On the exact ground state energy of Lieb and Wu, Prog. Theor. Phys. 45 (1971), 756-760. 